\title{
Uninterrupted power supply by integration of renewable energies, storage systems and smart energy management system in Kumasi-Ghana
}

\author{
M. Steglich ${ }^{1}$ and Johanna M.A. Myrzik ${ }^{1}$ \\ ${ }^{1}$ Institute of Energy Systems, Energy Efficiency and Energy Economics \\ TU Dortmund University \\ Emil-Figge-Straße 70, 44227 Dortmund (Germany) \\ Phone number: +49231 755-4302, e-mail: michael.steglich@tu-dortmund.de, johanna.myrzik@tu-dortmund.de
}

\begin{abstract}
Ghana currently has an unstable electrical power supply and load shedding is often used to ensure the stability of the grid. In this paper, a sustainable backup system with a smart energy management system to ensure a stable and uninterrupted power supply is presented. The system consists of two standalone inverters that connect two battery systems serving as energy storage units together with power generation from renewable energy sources i.e. photovoltaic systems and a biofuel generator. To achieve a continuous power supply of important loads in case of a power failure, a smart load management system has been developed and implemented. The load management system consists of load relays which are connected to one central control unit as well as to the loads in two buildings. The central control unit is also used to visualize all energy measurement data by using installed energy meters. The system is able to make important consumers energy self-sufficient and to supply them at least during $1.5 \mathrm{~h}$ in case of a grid failure.
\end{abstract}

\section{Key words}

Micro grids, smart grids, uninterrupted power supply, load shedding, renewable energy

\section{Introduction}

The electrical demand in Ghana grows faster than the available electricity generation in the country. This entails an unstable electrical power supply in Ghana. To avoid a complete power failure and in order to stabilize the power supply, the frequency and the voltage, it is common to shed the loads of districts or sometimes whole regions [1]. This can happen several times per day, so that there is no reliable and continuous power supply. Even important consumers like airports, hospitals and industries are affected.

This paper aims to present a concept for a sustainable uninterruptable power supply (UPS), integrating renewable energy sources, battery systems and a smart energy management system (EMS). The basis of the test infrastructure consists of the public grid as well as two buildings of the Kwame Nkrumah University of Science and Technology (KNUST) in Kumasi - Ghana. With the help of this concept consumers within the system are temporarily switched in islanding mode and load shedding is carried out in stages according to given priorities. The developed and implemented load shedding schemes prioritizes the loads according to importance such that 'high-priority' loads are not switched off during outages. This paper is organized as follows. In section 2, the test infrastructure and its elements as well as its functionality are explained. The energy management system and load shedding system are presented in section 3 . Finally, in section 4 conclusions are identified and an outlook is given.

\section{Setup and functionality of the renewable uninterrupted power supply}

In this chapter the specific components of the system and their functionality are explained.

\section{A. Components of the system}

The main components of the test infrastructure are two energy accumulators and one biofuel generator connected to the two inverters, seven PV-units and 25 energy meters. Additional components are load relays to enable the load shedding and an energy management system together with a central control unit. A general overview of the system is given in Figure 1.

\section{1) Energy accumulators}

Two stationary lead acid batteries with capacities of $730 \mathrm{Ah}$ and $1070 \mathrm{Ah}$ each with $48 \mathrm{~V}$ output voltage are used. The accumulator with the higher capacity is assigned to the building with the higher power consumption.

\section{2) PV systems}

On the roofs of both buildings are a total of seven PV-units each with a $12 \mathrm{kWp}$ installed power. Each $\mathrm{PV}$ - unit is connected to the respective grid of the building by a single-phase inverter. 


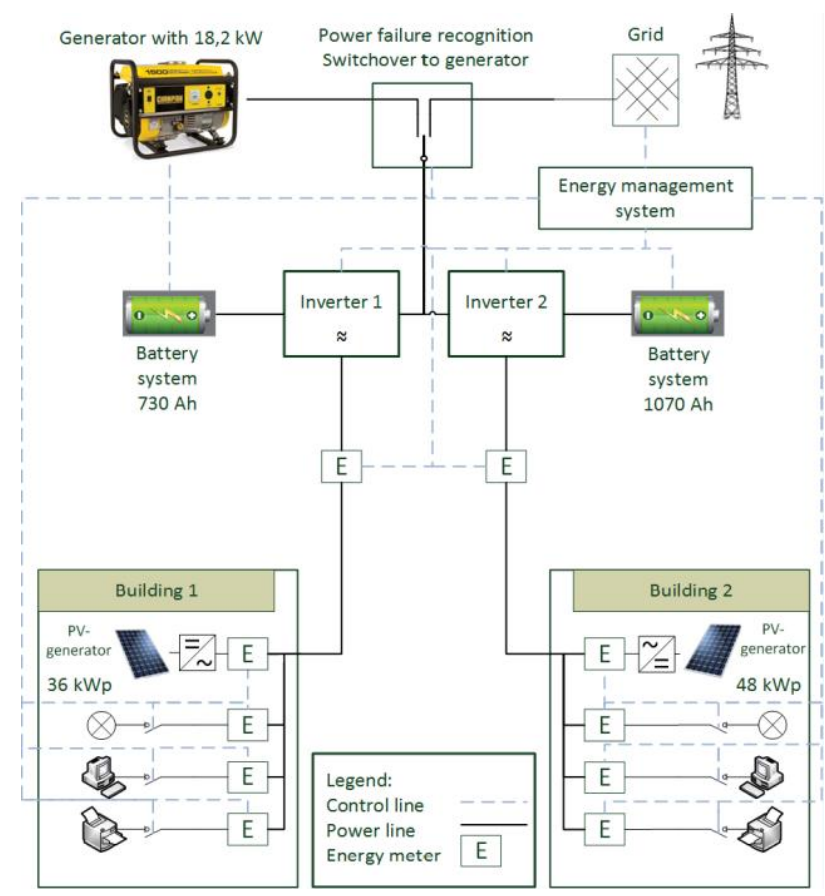

Figure 1: Setup of the test infrastructure

The PV's contribute to the power supply of the loads and the public grid.

\section{3) Inverters}

Each of the two buildings is connected to one threephase inverter, which is either switched between the islanding or grid connected mode. The chosen inverter are impervious to fine desert sand and high humidity, which predestines them for the use in Ghana [2]. The power limits of the two inverters are $18 \mathrm{kVA}$ and $13.8 \mathrm{kVA}$ provided that the loads are evenly distributed to the phases. This condition leads to a specific limitation per phase of $6 \mathrm{kVA}$ and $4.6 \mathrm{kVA}$. Exceeding this limit without the use of an EMS, causes the inverter to separate the whole phase from the system. Besides this, each inverter is connected to one accumulator and therefore works also as a battery charger.

\section{4) Biofuel generator}

The biofuel generator based on the Perkins engine feeds the loads of one building in the event of insufficient battery capacity or if activated manually. It has a nominal power of $18.2 \mathrm{~kW}$, a speed of $1500 \mathrm{rpm}$ and a consumption of 6.2 liter per hour. Oil from the local Jatropha plants in Ghana is used to fuel the generator. The EMS controls the activation of the generator and decides which building gets the energy.

\section{5) Energy meters}

The energy meters collect information about the input power of the PV systems and the consumed power of the loads. There are 25 single-phase and three-phase energy meters connected to the central control unit via a M-bus interface. The M-Bus is for the remote reading of the energy meters and consists of one master and several slaves. The central control unit serves as the master while the energy meters serve as the slaves. Thus, data logging of voltages, currents, reactive and active power is possible along with the central recording and retrieval of data. The energy meters are chosen based on the respective load limits. The central control unit is connected to the network by means of an Ethernet interface. This makes it possible to read the data via internet.

\section{6) Central control unit}

The central control unit named PCD (see Figure 2) is a free programmable, industrial control and automation device with onboard memory and communication interfaces [3]. It is a computer unit composed of several modules and it runs circularly and continuously through one control block. In this block, it is possible to implement control algorithms. With these algorithms it is possible to control and monitor the other components of the system by using the digital inputs and outputs of the PCD. The PCD gets the data from the installed energy meters by using the M-bus interface and contains the EMS and the load shedding management with further applications. As the control unit is fed from the isolated network it is not affected by the instability of the public grid and its operation is guaranteed.

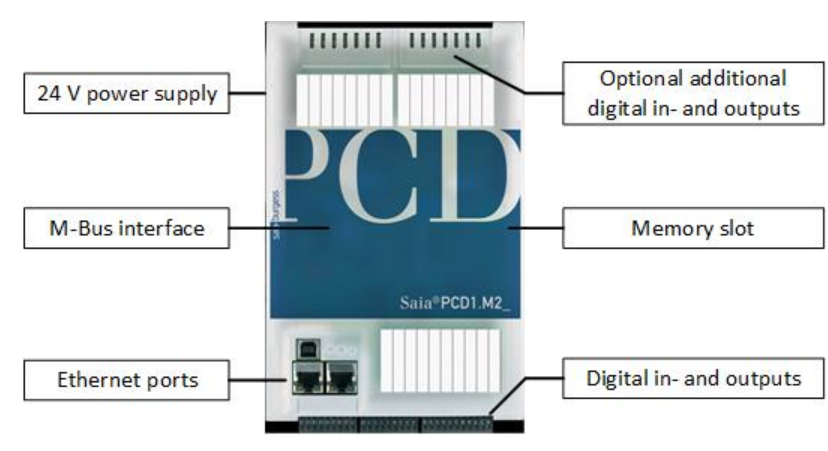

Figure 2: PCD components

\section{B. Functionality of the system}

The comprehensive uninterruptable power supply consists of two subsystems, each connected to one building. The biofuel generator provides energy for only one subsystem. The system works both during normal operation and during disturbance. If the public grid is active the battery systems are charged and the installed PV systems support the grid. If the PV systems generate more energy than the loads need, the surplus energy goes to the accumulators. Is the state of charge (SOC) of the accumulators $100 \%$, the PV systems are injecting into the public grid. In an event of disturbance, the inverter detects the outage immediately and use the battery system as energy source. In these instances the inverter creates an islanded grid and ensures the continuous power supply of the loads within the system. With the help of an additional relay, the inverter gets further information on whether the public grid or the generator is providing the energy. By means of this information, the inverter can prevent the surplus energy of the PV systems 
from being fed into the generator. Additionally, there are delay relays on the input side of the inverter, that allow a delay of at least five seconds during the switchover of the energy sources. This delay is a hardware specification of the inverters and needs to be taken into account. For example, the generator provides energy to the loads and the public grid is available again, then a direct switchover to the public grid is forbidden. Just after five seconds it is possible to connect to the public grid. If this delay is not taken into account it will lead to a malfunction of the inverter. Furthermore, the relays that are responsible for the switchover between the generator and grid to the inverters are connected with auxiliary relays. The auxiliary relays are connected to the central control unit and can communicate with it. In this way, the central control unit gets information about the contemporary energy source and it enables the monitoring of the functionality of every relay.

In case of a long term outage, the accumulators lose energy permanently. In case of defined SOC-values, the EMS reacts and the biofuel generator is switched on to provide enough energy for one subsystem. In the event of insufficient fuel or when the generator already provides energy for the other building, the accumulators steadily discharge until the critical SOC-value of $40 \%$. In this kind of situation, the protection against deep discharge intervenes and the EMS will disconnect the loads, which is explained in section 3-D.

In order to switch the loads on and off in a flexible way, there are 26 single-phase load relays installed in the distribution boards of the buildings. Additionally, to enable direct control by the PCD, control cables are laid and connected to the PCD. This enables the EMS to control and change the state of the loads depending on the situation. Different categories of priorities are defined for the loads. The most important loads are lights and sockets where important computers are connected. Less used rooms and sockets connected with, for example, photocopier, have a lower priority. It is however possible to easily change the priorities of the loads in a program on the PCD. This prioritized load shedding offers optimization potentials and added functions. The load shedding is explained more detailed in the next section.

\section{Energy Management System (EMS)}

The uninterruptable power supply is the basis of the emergency supply and the conceptual design of the EMS can improve its functionality. The main functions of the EMS are to ensure a stable and uninterrupted power supply such that the most important loads are supplied as long as possible. Furthermore, the EMS should be used to identify optimization potentials. As can be seen in Figure 3, the EMS is divided into several parts named data logging, load profiles, critical power check, load shedding and generator management. Figure 3 for instance shows that the generator management is only active in case of a public grid failure while the critical power check is on a higher level and is active respective of whether the public grid is active or not.

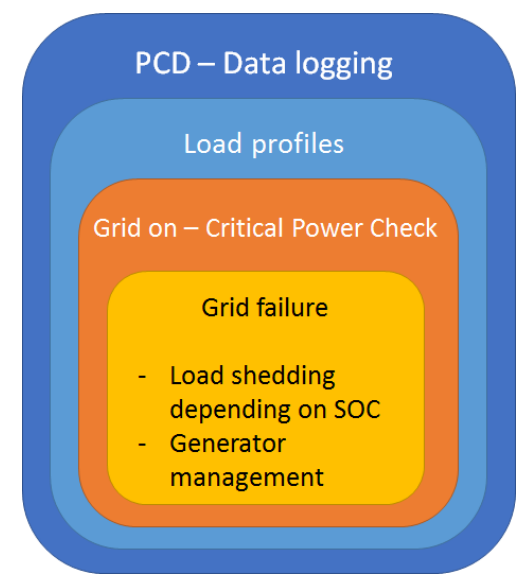

Figure 3: EMS components

\section{A. Data logging and visualization}

For data logging and visualization of the measurement data, a web interface has been implemented and 25 energy meters have been installed. The data is divided into two groups named 'devices' and 'grid'. Therefore, the control unit contains a multiplicity of data like outage times or the frequency of outages of the public grid and the active and reactive power of the loads and other parameters. The control unit enables reading and depiction of these parameters.

\section{B. Load profiles}

Several profiles can be loaded in the central control unit. As explained in section 2-B, the loads are assigned three different priority levels, which can be changed depending on the season and specific requirements of the UPS. In addition to this, the default profile in the control unit can be temporarily overwritten and subsequently reset as required. Table 1 shows an example of defined load priorities during lecture period and the semester break, which can be changed easily by changing the load profile. This characteristic allows the use of different profiles, which consider holidays or semester breaks. A typical profile could be for instance to give the lecture rooms a lower priority during semester break than during the semester while the priority of the offices are increased. Another example would be an important event, meeting or presentation in a certain room. The use of a respective profile guarantees the longest possible operation time for every load in this room by assignment of the highest priority.

Table I. - Load priority allocations

\begin{tabular}{|l|c|c|c|}
\hline \multicolumn{1}{|c|}{ Room } & Load & $\begin{array}{c}\text { Lecture } \\
\text { period }\end{array}$ & $\begin{array}{c}\text { Semester } \\
\text { break }\end{array}$ \\
\hline & & Prioritization & Prioritization \\
\hline Lecture Hall & Light & High & Medium \\
\hline Lecture Hall & Sockets & Medium & Low \\
\hline Storeroom & All & Low & Low \\
\hline Employee office & Light & High & High \\
\hline Employee office & Sockets & Low & Medium \\
\hline Student office & Light & High & High \\
\hline Student office & Sockets & Medium & Low \\
\hline
\end{tabular}




\section{Critical power check}

To protect the power lines of the island system against overload, a power check is implemented, which is active during both normal operation of the grid and during disturbances. As already mentioned in section 2-A.3, the respective power limitations of the two inverters; $18 \mathrm{kVA}$ and $13.8 \mathrm{kVA}$ lead to a maximum power per phase of $6 \mathrm{kVA}$ and $4.6 \mathrm{kVA}$. In order that the inverters do not deactivate the whole system by exceedance, the control unit does a critical power check. With the help of the energy meters, the control unit knows the actual power per phase. In the event of exceedance of the actual power of one phase, the PCD disconnects the load with the lowest priority of the affected phase. If the power limit is still overstepped or get overstepped later on, the next priority of loads will be shed. This avoids a direct and complete shutdown of the system and makes it more efficient.

\section{Load shedding management}

The load relays are connected to the central control unit and the main part of the load shedding management. The PCD gets information about the SOC range of values of the accumulators from the inverters. This makes it possible for the PCD to make decisions. During outages of the public grid and depending on the capacity of the accumulators, the EMS which separates the loads with low priority from the island grid, leads to longer supply of the loads with higher priority. The EMS currently distinguishes three levels of priority: 'low', 'medium' and 'high'. The central control unit is connected with control lines to several distribution boxes in both buildings and is responsible for the load shedding. This enables the switching on or off of specific relays and the attached loads. At the moment, loads with high energy consumption and low priority like air conditioner are shed at first, whereas projectors in lecture rooms or PCs have a higher priority and are supported longer. If the SOC-value reaches $40 \%$ or less, the load shedding management separates every load from the system to ensure protection against deep discharge and to extend the life span of the batteries.

\section{E. Generator management}

The biofuel generator can be activated by the PCD depending on the SOC-value or due to a direct command of the control unit. For this purpose, a control line is installed between the PCD and the generator starter. The activated generator feeds the loads and charges the battery of the building. Subsequently, the shed loads are connected again. Due to the restriction that the generator can only feed one building, the EMS decides which building is supplied. To make this decision, the buildings get different priorities. As long as the SOC of the building with the higher priority is too low, the generator provides energy to this building from this moment on regardless of whether the generator already feeds the other building or not. This setting can be changed temporarily to offer higher flexibility. An internal timer is also part of the PCD and protects the generator against too fast reconnection and possible hysteresis loops. This is necessary because the generator needs a cool-down time of at least 3 minutes after deactivation. Too fast reactivation can occur in case of frequent starts and shutdowns of the public grid and change to emergency power mode during the cool-down time of the generator. This needs to be avoided because to rapid reactivation would stress the generator too much and can damage it. An internal timer in the PCD avoids this problem and protects the generator from too fast reactivation.

\section{Conclusion and outlook}

In this paper, a sustainable uninterruptable power supply with renewable energy sources, an energy management system and load management system has been presented based on a field test at the campus of the KNUST. Because of the communication and interaction between the individual components of this system, it resembles a smart grid [4]. The central control unit enables the flexible switching on or off of the loads depending on adjustable priorities and the specific situation. With the use of PV generators, a biofuel generator and accumulators the system can operate in an islanding mode during grid outages. Through the inverter, the uninterruptable power supply is guaranteed. Furthermore, there are several strategies to protect the batteries against deep discharge and the generator and inverter against damages. The critical power check avoids the complete shutdown of the system in the event of an overload and facilitates the supply of the most important loads. Presettable load profiles with different load priorities enable the consideration of seasonal effects and make the system more flexible. Finally, the central connection of every energy meter provides the possibility to measure electrical parameters like for example the energy consumption or the utilization factor of the inverters.

Identification of optimization potentials takes several months of system operation and data measurement. Imaginable potentials are situated in the fields of an improved load prioritization, the design of more preinstalled load profiles and a better battery and generator management. Right now the central control unit gets the information whether the SOC of the battery is above $70 \%$, between $40 \%$ and $70 \%$ or under $40 \%$. A huge improvement would be, if the central control unit knows the exact SOC. In this case for instance more level of prioritizations can be created and the load shedding could be improved significantly.

\section{References}

[1] Electricity Company of Ghana LTD, [online] http://www.ecgonline.info/index.php/customer-care/outagecenter/view-outages.html [25.10.17]

[2] SMA, [online] https://www.sma.de/en/products/batteryinverters/sunny-island-60h-80h.html [25.10.17]

[3] SAIA Burgess Control, [online] https://www.sbcsupport.com/en/product-category/programmablecontroller/pcd1-cpus-in-flat-design/pcd1m2160/ [25.10.17]

[4] Hassan Farhangi, "The path of the smart grid", in Proc. 2009 IEEE Power \& Energie Society, pp. 19-22 